\title{
Evaluation of Image Quality of Plain Abdominal Radiographs at University of Maiduguri Teaching Hospital, North-Eastern Nigeria.
}

\author{
A.B Shettima, FA Malgwi, N.M Gunda, IC Nwobi, FL Gajerima, \\ A. Abubakar, MB Kurama. \\ Department of Medical Radiography, University of Maiduguri, Borno State Nigeria. \\ Department of Medical Radiography University of Maiduguri, Borno State Nigeria.
}

\begin{abstract}
Objectives: To determine the quality of abdominal radiographs, and common factors that affect them.

Method: A retrospective non experimental study was conducted on abdominal radiographs produced at UMTH between 2010 and 2015, assessing its quality using European commission guidelines and radiographic technical parameters as criteria.

Data Analysis: Descriptive statistics such as frequency and percentages were generated from the result and the data was analyzed using a trial version of statistical package for social sciences (SPSS Version 16.0).

Results: A total of 260 adult abdominal radiographs were evaluated of which $79.2 \%(n=206)$ were supine and $20.8 \%(n=54)$ were erect abdominal radiographs. Out of the radiographs studied, $21.2 \%(n=55)$ were obtained using computed radiography system while $78.8 \%(n=205)$ were obtained using film-screen system. Based on the radiographic technical parameters only $50.6 \%(n=132)$ were of optimal quality. While $49.4 \%(n=128)$ of the radiographs were sub-optimal quality. The overall image quality score for abdominal radiographs was $3.8 \%(n=10)$ optimal and $35.8 \%(n=93)$ very poor. This is due to poor radiographic technique and ineffective quality control protocol.
\end{abstract}

Conclusion: The Abdominal radiographs were of very poor quality in UMTH within the period studied.

Keywords: image quality, Abdomen, Radiographs.

\section{Introduction}

Image quality is a characteristic of an image that measures the perceived image degradation. Image quality is a broad term which can mean different thing to different people. For instance, a Radiologist when viewing a radiograph may be interested primarily in the diagnostic value of an image while Radiographer may focus on how well the images represent the anatomy [1].

Image quality of a radiograph is determined by the imaging process and the characteristics of the equipment and the imaging system variables selected by the operator. It is not a single factor but is a composite of contrast, blur, noise, artifact [2]. Medical image quality is also related to the subjective interpretation of visual data. It represents the clinical information contained in the image. It is more important that the observer interpret the image appropriately than whether the appearance of the image is pleasing to the eye. The ideal set of parameters to describe image quality should measure the effectiveness with which an image can be used for its intended purpose [3].

Regular quality assurance (QA) testing should be carried out for sustaining good practice in diagnostic imaging is important because it reduces practices that could affect expected patient outcomes and therefore justify the process of patient irradiation. It aims to ensure that every image produce is of diagnostic value in order to satisfy set of clinical targets [4].

Assessment of image quality (using physical or visual techniques) is a low order task as the results are commonly used to infer whether an image is fit for its intended purpose. Studies of image quality are essential for optimization of the radiography processes in any clinical settings [5].

Guidelines have been set up by the European Commission (EC) for assessing the basic aspects of quality for clinical radiographic images dependent on techniques and imaging performance [3].

Generally, high image quality provides maximum information, improve the chance for correct diagnosis and ultimately contribute to quality patient care.

Plain abdominal radiography can be readily obtained within a short period of time to help a physician arrive at a correct diagnosis [6], thus, it is among the commonly requested investigation for patients with abdominal symptoms, particularly in the Accident and Emergency (A \& E) departments [7]. They can also be used in assessing levels of patient preparation for contrast based examinations of the gastrointestinal tract (GIT) [8]. 
Plain abdominal $\mathrm{x}$-ray involves exposing part of the body (Abdomen) to a dose of ionizing radiation to provide the image of the organs within the abdomen and the common projections are the Antero-posterior Supine and Erect. Standard abdominal radiograph should include the area from diaphragm to the pubic symphysis [9]. Abdominal x-ray should be performed only for a valid medical reason and with the minimum radiation dose necessary to achieve a diagnostic study [9].

\section{Methods}

A retrospective survey study was conducted. Abdominal radiographs, obtained between January 2010 and December 2015, in the radiology department of University of Maiduguri Teaching Hospital (UMTH) were reviewed. Ethical clearance to conduct the research was obtained from the research committee of the institution. Quality assessment was done in two parts: first was identification of technical parameters such as complete patient identity, correct placement of anatomical marker, silver lining showing evidence of adequate Collimation, symmetry of anatomical parts showing evidence of no rotation of patient to one side, Blurring (motion) Penetration and Artifact

Second was assessment of image quality from the guidelines recommended by the European commission were adopted [10] these were:

Area of coverage, visualization of kidney outline, visualization of psoas major muscle, visually sharp reproduction of bones and image centralization.

Radiographs were scored optimal quality if all the criteria listed were observed. A score of sub-optimal quality was given to films with three or more, but less than the six listed criteria, while Poor quality was given for films with less than three listed criteria.

\section{Results}

A total of 260 adult supine $(n=206)$ and erect $(n=54)$ abdominal radiographs of both male $(n=160)$ and female $(n=100)$ were evaluated. A total of $21.2 \%(n=55)$ of the radiographs were obtained using computed radiography system while $78.8 \%$ (205) were obtained using film-screen cassette.

Results of the evaluation of technical parameters shows that, Presence of Anatomical marker was the highest fulfilled technical parameter as evidenced on $91.5 \%(\mathrm{n}=238)$ of the radiographs. This is followed by motional blurring having $68.1 \%(n=177)$. Penetration was adequate in $55.8 \%(n=145)$ of the radiographs, inadequate in $14.2 \%(n=37)$ and poor in $30.0 \%(n=78)$. A total of $47.7 \%(n=145)$ of the radiographs had no evidence of rotation while $52.3 \%(n=136)$ showed evidence of rotation. Artifacts were present in $40.8 \%(n=106)$ of the radiographs while $59.2 \%(n=154)$ had no artifacts. Patient identification was adequate in $21.9 \%(n=57)$, inadequate in $66.9 \%(\mathrm{n}=174)$, and poor in $11.2 \%(\mathrm{n}=29)$. The least fulfilled technical parameter was beam collimation with only $18.8 \%(\mathrm{n}=49)$ recorded as adequate, $6.9 \%(\mathrm{n}=18)$ as inadequate and $74.2 \%(\mathrm{n}=193)$ as poor/none collimation. The overall radiographic technical parameter shows that $50.6 \%(\mathrm{n}=132)$ of the abdominal radiographs were of optimal quality while $49.4 \%(n=128)$ were of sub optimal quality (Table 1.0$)$.

On the other hand, results of Assessment of image quality of abdominal radiograph showed that; sharp visualization of bone $78.1 \%(\mathrm{n}=203)$ as a parameter of image quality has the highest percentage. This is followed by image centralization with $70.8 \%(n=184)$ and area of coverage $64.2 \%(n=167)$.

Visualization of Kidney outlines $9.6 \%$ is the parameter of image quality with the least percentage. Psoas muscle and Liver outlines were visible in $16.9 \%(n=44) 19.2 \%(n=50)$ and invisible in $83.1 \%(n=216)$ $80.8 \%(\mathrm{n}=210)$ respectively (figure 1.0$)$.

Generally, 3.8\% (n=10) of the radiographs were found to be optimal indicating that they fulfilled all the six criteria, $60.4 \%(n=157)$ were sub-optimal indicating that the radiographs fulfilled 3 above but less than six criteria while $35.8 \%(n=93)$ were poor/none which indicates that the radiographs failed to satisfy a criteria (scoring $\leq 2$ criteria).

Table 1.0 Result of Assessed radiographic technical parameters.

\begin{tabular}{|c|c|c|c|c|c|c|c|}
\hline \multirow{3}{*}{$\begin{array}{l}\mathbf{S} / \mathbf{n} \\
1\end{array}$} & \multirow{3}{*}{\begin{tabular}{|l} 
Parameters \\
Patient ID \\
\end{tabular}} & \multicolumn{6}{|c|}{ Frequency \& Percentage (\%) of films } \\
\hline & & \multicolumn{2}{|c|}{ Adequate } & \multicolumn{2}{|c|}{ Not adequate } & \multicolumn{2}{|c|}{ Poor/none } \\
\hline & & 57 & $21.9 \%$ & 174 & $66.9 \%$ & 29 & $11.2 \%$ \\
\hline 2 & Anat. Marker & 238 & $91.5 \%$ & - & - & 22 & $8.5 \%$ \\
\hline 3 & Collimation & 49 & $18.8 \%$ & 18 & $6.9 \%$ & 193 & $74.2 \%$ \\
\hline 4 & Rotation & 124 & $47.7 \%$ & 136 & $52.3 \%$ & - & - \\
\hline 5 & Blurring (motion) & 177 & $68.1 \%$ & 83 & $31.9 \%$ & - & - \\
\hline \multirow[t]{2}{*}{6} & Penetration & 145 & $55.8 \%$ & 37 & $14.2 \%$ & 78 & $30.0 \%$ \\
\hline & & \multicolumn{2}{|c|}{ Present } & \multicolumn{2}{|c|}{ Not present } & & \\
\hline \multirow[t]{2}{*}{7} & Artifacts & 106 & $40.8 \%$ & 154 & $59.2 \%$ & - & - \\
\hline & & \multicolumn{2}{|c|}{ CR cassette } & \multicolumn{2}{|c|}{ FS cassette } & & \\
\hline 8 & Image receptor & 55 & $21.2 \%$ & 205 & $78.8 \%$ & - & - \\
\hline
\end{tabular}




\begin{tabular}{|c|c|c|c|c|c|c|c|}
\hline & & & & & & & \\
\hline & & \multicolumn{2}{|c|}{ Supine } & \multicolumn{2}{|c|}{ Erect } & & \\
\hline 9 & projection & 206 & $79.2 \%$ & 54 & $20.8 \%$ & - & - \\
\hline
\end{tabular}

Patient ID= patient identification $\quad F S=$ film screen

Anat. Marker $=$ anatomical marker

$\mathrm{CR}=$ computed radiograph

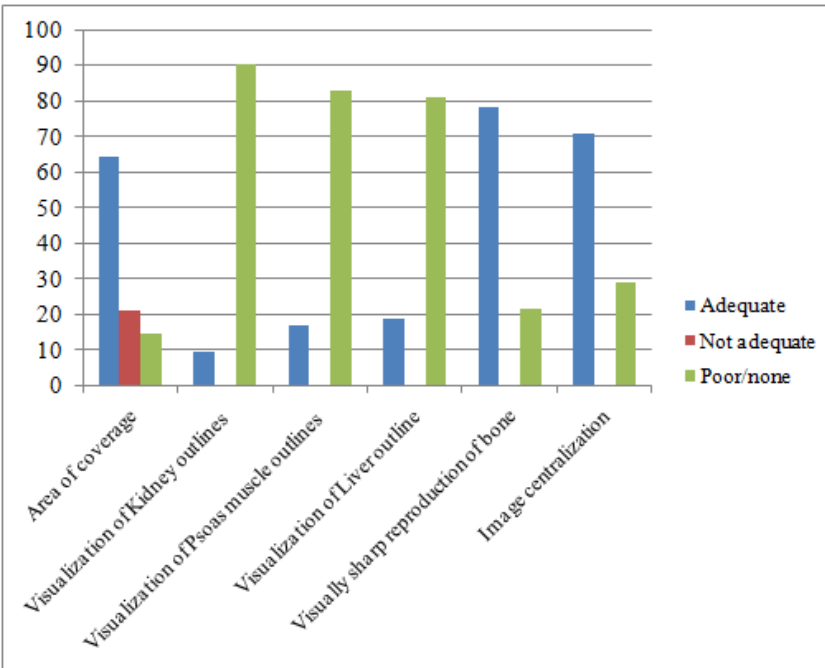

Figure 1.0: Image quality criteria of plain abdominal radiographs

\section{Discussion}

The usefulness of diagnostic radiographs depends on the quality of the produced image [11]. Image quality is the integrated set of perception of the overall degree of excellence of an image [12]. The determination of quality should be conducted by measuring the performance of an observer for a diagnostic task. The outcome from the interpretation of a medical image is a clinical diagnosis and accuracy of that inference about the status of the human anatomy is imperative for successful patient treatment [13].

In this study, the abdominal radiographs were evaluated based on the radiographic technical parameters and image quality criteria defined by commission of European Communities (EC). Out of the 260 abdominal radiographs studied, only $50.6 \%(n=132)$ were adequate, these are those that met all the listed technical parameters while $49.4 \%(n=128)$ of the radiographs were inadequate. This is in line with a study conducted by inah et al., [14] in which 194 radiographs were reviewed. They reported that only 57\% ( $=108)$ of the radiographs were adequate while $43 \%(n=83)$ of the radiographs had technical faults.

Collimation is one of the technical factors studied. Previous studies by Inah et al., [14] reported that only $1.1 \%(\mathrm{n}=2)$ of the radiographs had adequate collimation while $98.9 \%(\mathrm{n}=192)$ were inadequate. Egbe $e t$ al.,[7] also found that only $47.8 \%(\mathrm{n}=100)$ had adequate while $52.2 \%(\mathrm{n}=110)$ were not adequate. Collimation was rated as inadequate in about $50 \%(n=22)$ of the images [15]. This varies from the present study which found that beam collimation is the most common factor that affect the quality of the radiographs with only $18.8 \%$ $(n=49)$ having adequate collimation while $74.2 \%(n=193)$ had poor collimation. This may be due to misalignment of the beam or improper collimation during procedures which reveals poor adherence to radiation protection rules in the hospital. Adoption of proper beam collimation will reduce the potential harmful effect of ionizing radiation and improve the quality.

Another technical factor assessed in this study was the patient rotation. Egbe et al., [16] found that $40 \%$ $(n=84)$ of the radiographs had rotation due to poor patient positioning, Muhammed et al.,[17] reported 16\%, Inah et al., [14] reported 47.7\% (93) and Oliveira et al.,[15] reported 20\% (9). In this study we found that $52.3 \%$ (136) of the radiographs had rotation which is not in agreement with the above reported values. Moreover the value from this research is higher than their findings; so may be differences in the condition of patient in the different studies, patient's comfort and ability to maintain position during examination.

Artifacts were present in $40.8 \%(n=106)$ of the radiographs studied and most of the artifacts found in this study were due to improper fixation (thiosulphide deposit) affecting most of the radiographs produced in 2012 and 2015. This should be in agreement with Jeanne et al.,[18] who discovered that the major causes of poor quality images were due to the existence of artifacts with $75 \%$ and $100 \%$ of the radiographs being affected in hospital 1 and hospital 3 respectively. 
With regard to the image quality criteria assessed,kidney outlines were visualized in $9.6 \%(n=25)$ of the radiographs while psoas major muscle outlines was visible in $16.9 \%(\mathrm{n}=44)$. This is most likely due to the selection of improper exposure factors.

Another criteria studied was the area of coverage. In a study carried out by panthagani et al.,[19]. It was reported that $56 \%$ of the radiographs were fairly covered while $28 \%$ were poorly covered. This is higher than the present study which found $21.2 \%$ were fairly covered and $14.6 \%$ were poorly covered.

In the present study we found that the overall image quality score for abdominal radiographs was $3.8 \%(\mathrm{n}=10)$ adequate, $60.4 \%(\mathrm{n}=157)$ not adequate and $35.8 \%(\mathrm{n}=93)$ very poor this could probably be due to old machines. This is in contrast with the studies conducted by Egbe et al., [16] who reported 50.9\% adequate and $6.61 \%$ poor and most probably repeated or rejected. In the same vein, Muhammed et al., [17] found that more than $40 \%$ of the radiographs were not adequate majorly due to overexposure. Ahmed et al., [20] on the other hand found that up to $70.4 \%$ of the radiographs were adequate while $19.3 \%$ were not adequate. Jameela et al., [21] reported that the observed high percentage of poor image quality is likely due to an absence of quality assurance (QA) program in the study areas. We strongly believe that the disparity in the result of this study from other studies is due to variations in adopted protocols and techniques, personnel training and expertise as well as equipment type and age of the equipment.

\section{Conclusion}

Abdominal radiographs produced in Univeristy of Maiduguri teaching hospital north eastern Nigeria reveals technically good individual criteria image performance but possess overall sub-optimal quality. A lot of improvement is needed in the area of study and this will largely be achieved by the implementations of QA programmes.

\section{Recommendation}

The result obtained in this study discovered that the factors attributed to low image quality include poor radiographic technique and ineffective quality control protocols. This indicates the need for improvement of quality assurance programme and optimization of radiographic procedure to enhance image performance in the study area.

\section{Reference}

[1]. International Commission on Radiological Protection, Radiological and Safety in Medicine, Publication 73, Annals of the ICRP, 26/2: Oxford, UK; Pergamon press. (1996)

[2]. Sprawls P., Physical Principles of Medical Imaging $2^{\text {nd }}$ ed.[online]. Available at: http//www.sprawls.org/resources. (2014),

[3]. Martin, CJ. Optimization In General Radiography: Biomedical Imaging and Intervention Journal 3(2),1-14. (2006).

[4]. International Atomic Energy Agency, Optimization of the Radiological Production of Patients, final report of a coordinated research project in Africa, Asia and Estern Europe;IAEA-TECDOC-1423. (2004).

[5]. Peter, H. and Luis, L., Radiation Dose and Image Quality Optimization in Medical Imaging. Lisbon, Portugal. (2014).

[6]. SmittJE. and Hall EJ., Diagnostic Imaging Pathways Indications Of Plain Abdominal X-ray In Emergency Department: Emergency Medicine Journal, 26(3),160-3. (2009).

[7]. Egbe, NO, Eduwem, DU, and Ikamaise, VC, Investigation of Image Quality of Plain Abdominal Radiographs in Three Nigeria Hospitals: Biomedical Imaging and Intervention Journal.3 (4), 39. (2007).

[8]. Wadman M Syk I, Elmstah B et al, Abdominal Plain Film Findings In Acute Ischemic Bowel Disease Differ With Age. Acta Radio journal, 47(3). 238-243. (2006).

[9]. American College of Radiology, ACR Practice Parameter for the Performance of Abdominal Radiography. Available at: http//www.arc.org/ /media. (2014).

[10]. European commission. European Guidelines on Quality criteria for Diagnostic Radiographic images. Luxemburg, EUR 16260 EN. (1996).

[11]. Jessen, KA, Balancing Between Image Quality and Dose In Diagnostic Radiology. European Radiology Supplement, 14(1), 9-18. , (2004),

[12]. Marini, F. et al., Image Quality in Multimedia Application. Available At: http//www.ivl.disco.unimib.it. (2015).

[13]. Kundel, HL.et al., Perception and Representation Of Medical Images. SPIE: Image Processing, 1898: 2-12. (1993).

[14]. Inah, GB, Akintomide, AO, and Edim, UU, A Study Of Pelvic Radiography Image Quality In A Nigerian Teaching Hospital Based On The Commission Of European Communities Criteria. The South African Radiography, 15(2), 15-18. (2013).

[15]. Oliveira, AC. et al., Visual Grading Analysis of Image Quality In Pediatric Abdominal Images. European Society of Radiology; 124. (2016)

[16]. Egbe, N.O., Chiaghanam, N.O., Azogor, W.E., and Inyang, S.O., A Baseline Study of Entrance Dose and Image Quality for Lumber Spine Radiography in Calabar, Nigeria. Radiography 15, 306-312. (2013)

[17]. Muhammed, JK. et al., Evaluation of Image Quality and Patient Dose in Conventional Radiography Examinations in Radiology Centres In Sistan And Baluchestan, Iran. Indian Journal Of science And Technology, 4(11), 1429-1433. (2011).

[18]. Jeanne MR.et al., Survey of Image Quality and Patient Dose in Simple Radiographic Examinations in Madagascar. HEP-MAP 09, 1-5. (2009).

[19]. Pathagani J., Oshea, E, Howie A. and Patel NG. Assessment of the Image Quality and Indications for Plain Abdominal Radiography: European Society of Radiology. Available at: http//www.myESR.org. (2015).

[20]. Ahmed, NA.et al., Patient Dose and Image Quality Evaluation In Common Radiographic Examinations in Sudan. Research Gate,13. (2009).

[21]. Muhogora WE. et al., Patient doses in radiographic examination in 12 countries in Asia, Africa and Eastern Europe: initial results from IAEA projects, AJR Am J roentgenol 190(6):1453-61. (2008). 